\title{
Triple Smoking Cessation Therapy with Varenicline, Nicotine Patch and Nicotine Lozenge: A Pilot Study to Assess Tolerability, Satisfaction and End-of-Treatment Quit Rates
}

\author{
Kristin M. Berg, ${ }^{1,2,3}$ Douglas E. Jorenby, ${ }^{2}$ Timothy B. Baker, ${ }^{2}$ and Michael C. Fiore ${ }^{2}$ \\ ${ }^{1}$ Division of General Internal Medicine, Department of Medicine, University of Wisconsin School of Medicine and Public Health, \\ Madison, Wisconsin \\ ${ }^{2}$ Center for Tobacco Research and Intervention, University of Wisconsin School of Medicine and Public Health, Madison, \\ Wisconsin \\ 3 Primary Care Research Fellowship, Department of Family Medicine and Community Health, University of Wisconsin School of \\ Medicine and Public Health, Madison, Wisconsin
}

\begin{abstract}
ntroduction: The majority of attempts to stop smoking end in failure. One way to improve success may be to explore different combinations of existing cessation medications.

Aims: This observational study examined 'triple therapy' (varenicline + nicotine patch + nicotine lozenge) in 36 smokers trying to quit.

Methods: A 12-week, observational study exploring tolerability, via adverse events (AEs) elicited at each of nine phone assessments. Secondary outcomes included satisfaction rates, medication changes and self-reported quit rates at week 12 .

Results: Thirty five of thirty six participants reported at least one AE. Insomnia (75\%), abnormal dreams $(72 \%)$ and nausea (64\%) were most common. Most were mild to moderate. No deaths, hospitalisations, cardiovascular events or suicidality were reported. Six participants $(17 \%)$ decreased the dose of at least one medication, $5(14 \%)$ decreased the dose then discontinued at least one medication and $13(36 \%)$ discontinued at least one medication without trying a lesser dose. Participants were highly satisfied with their medications, and 58\% reported quitting at 12 weeks, with $38 \%$ reporting prolonged abstinence.

Conclusions: Despite high rates of AEs and medication changes, high rates of satisfaction and selfreported quitting, with no serious AEs, were observed with triple therapy. Additional data on tolerability and efficacy are needed.
\end{abstract}

Trial Registration: Clinicaltrials.gov number NCT02681510.

\section{Introduction}

For both healthcare providers and their patients who smoke, a lack of new evidence-based treatment options may result in frustration, reduce use of evidence-based treatment and impede cessation success. The most effective FDA-approved medications include combination nicotine replacement therapy (cNRT) and varenicline, but these yield abstinence rates of only $33-37 \%$ at 6 months (Fiore, Jaen and Baker, 2008). Moreover, no new medications have been approved by the FDA since varenicline in 2006. As one strategy to boost smoking cessation rates, new combinations of currently approved therapies have been evaluated, such as varenicline plus NRT (Koegelenberg et al., 2014). Varenicline is a partial agonist of $\alpha 4 \beta 2$ nicotinic acetylcholine receptors, but may not saturate receptors (Ebbert, Hays, \& Hurt, 2010), and other pathways are likely involved in the neuronal response to nicotine (Benowitz, 2010). It has been postulated that adding NRT to varenicline could more fully saturate receptors and target accessory pathways (Chang et al., 2015; Ebbert, Hays, \& Hurt, 2010), potentially improving abstinence rates. 
Three randomised controlled trials have compared varenicline plus nicotine patches (Hajek, Smith, Dhanji, \& McRobbie, 2013; Koegelenberg et al., 2014; Ramon, Morchon, Baena, \& Masuet-Aumatell, 2014) versus varenicline plus placebo patches. Two of these trials (Hajek, Smith, Dhanji, \& McRobbie, 2013; Ramon, Morchon, Baena, \& Masuet-Aumatell, 2014) demonstrated no statistically significant abstinence benefit with the combination therapy at 12 weeks, but the largest trial (Koegelenberg et al., 2014), which also utilised pre-quit nicotine patch, demonstrated that combination therapy produced substantially higher quit rates at 12 weeks and 6 months. A subsequent metaanalysis of these three trials demonstrated a significant increase in abstinence rates at the end of treatment and post-treatment follow-up (Chang et al., 2015), but the results were not statistically significant if the third trial (Koegelenberg et al., 2014) was removed. There has been one evaluation of 'triple therapy' (varenicline plus nicotine patches plus short-acting NRT) versus cNRT alone (Ebbert, Burke, Hays, \& Hurt, 2009), but this was a retrospective review of 239 patients, had variable treatment regimens across patients, and occurred in an inpatient nicotine treatment centre. Self-reported abstinence rates at 6 months were $54 \%$ for the triple therapy group, and $59 \%$ for the cNRT group (Ebbert, Burke, Hays, \& Hurt, 2009). While these abstinence rates do not support the use of triple therapy, features of the study may limit the external validity of the results. For instance, the study was not randomised, and the inpatient venue may have greatly enhanced abstinence in both groups and masked the differential effects of the medication conditions.

One factor that could limit the use and acceptability of triple therapy is tolerability. The use of three medications could certainly produce additive or synergistic effects that increase the number or severity of adverse events (AEs). Of the randomised controlled trials of varenicline plus nicotine patch, only skin reactions (Koegelenberg et al., 2014) and abnormal dreams (Hajek, Smith, Dhanji, \& McRobbie, 2013) were noted to be more prevalent in the combination group than in the varenicline only group. Ramon, Morchon, Baena, \& Masuet-Aumatell (2014) did not find significant differences in AEs, nor did Chang et al. (2015) in their meta-analysis. The retrospective evaluation of triple therapy recipients (Ebbert, Burke, Hays, \& Hurt, 2009) demonstrated that this group had fewer overall AEs (39\% of patients reporting at least one $\mathrm{AE}$ ) versus the cNRT group (59\% reporting at least one AE). However, unpublished data reported in the Pfizer varenicline package insert noted that co-administration of varenicline plus nicotine patch was associated with an increase in AEs, and $36 \%$ of patients using combination therapy prematurely discontinued medications, compared to $6 \%$ of patients treated with NRT plus placebo (Pfizer Laboratories Div Pfizer Inc, 2016).

Given the potential, but unclear, benefits of adding NRT to varenicline, the methodologic variations amongst previous studies, and the lack of prospective trials investi- gating triple therapy, we undertook an observational study examining triple therapy in 36 smokers attempting to quit. Our primary outcome of interest was to determine tolerability of triple therapy. Secondary outcomes included rates of medication changes, participant satisfaction, selfreported point-prevalence and prolonged quit rates at 12 weeks. We hypothesised that there would be more AEs from triple therapy, but that participants would express high levels of satisfaction. We hypothesised that we would see a higher rate of abstinence with triple therapy compared with prior studies using varenicline or cNRT alone.

\section{Methods}

This study was approved by the University of Wisconsin Health Sciences Institutional Review Board, and registered with clinicaltrials.gov, NCT02681510.

\section{Design}

This was an observational study designed to assess tolerance and safety of triple therapy for smoking cessation using varenicline, nicotine patch and nicotine mini-lozenge. Participants were recruited via Facebook ads contextually tagged to appear only to adults in the Madison, WI area who had 'liked' or posted about smoking. Sample size goal was 40 participants. The sample size of 40 was chosen because we believed it would provide a meaningful estimate of $\mathrm{AE}$ rates at a reasonable expenditure of time and costs. Please see (Figure 1) for the STROBE diagram and study flow.

Inclusion criteria were age $>17$ years; $>=$ five cigarettes/day for the previous 6 months; alveolar $\mathrm{CO}>=$ $6 \mathrm{ppm}$; able to read, write and speak English; planning to remain in the intervention catchment area for at least 4 months; not currently taking bupropion or varenicline; if the participant was currently using NRT, s/he agreed to use only study medication for the duration of the study; free of medical contraindications to NRT and varenicline (e.g., allergic reactions); and, if a participant was a woman of childbearing potential, using an approved method of birth control during treatment. Exclusion criteria were current diagnosis of, or treatment for, psychosis or bipolar disorder; suicidal ideation within the past 12 months; any history of suicide attempt; significant hepatic or renal impairment; use of any investigational drugs in the previous 30 days.

\section{Procedures}

Potential participants who provided contact information via the Facebook ads were called and provided a detailed description of the study and participation requirements. Inclusion and exclusion criteria were assessed, and those who met criteria were scheduled for a baseline study visit. At the initial study visit, potential participants provided written informed consent and completed surveys about nicotine dependence and withdrawal, smoking history and use of non-combustible tobacco products 


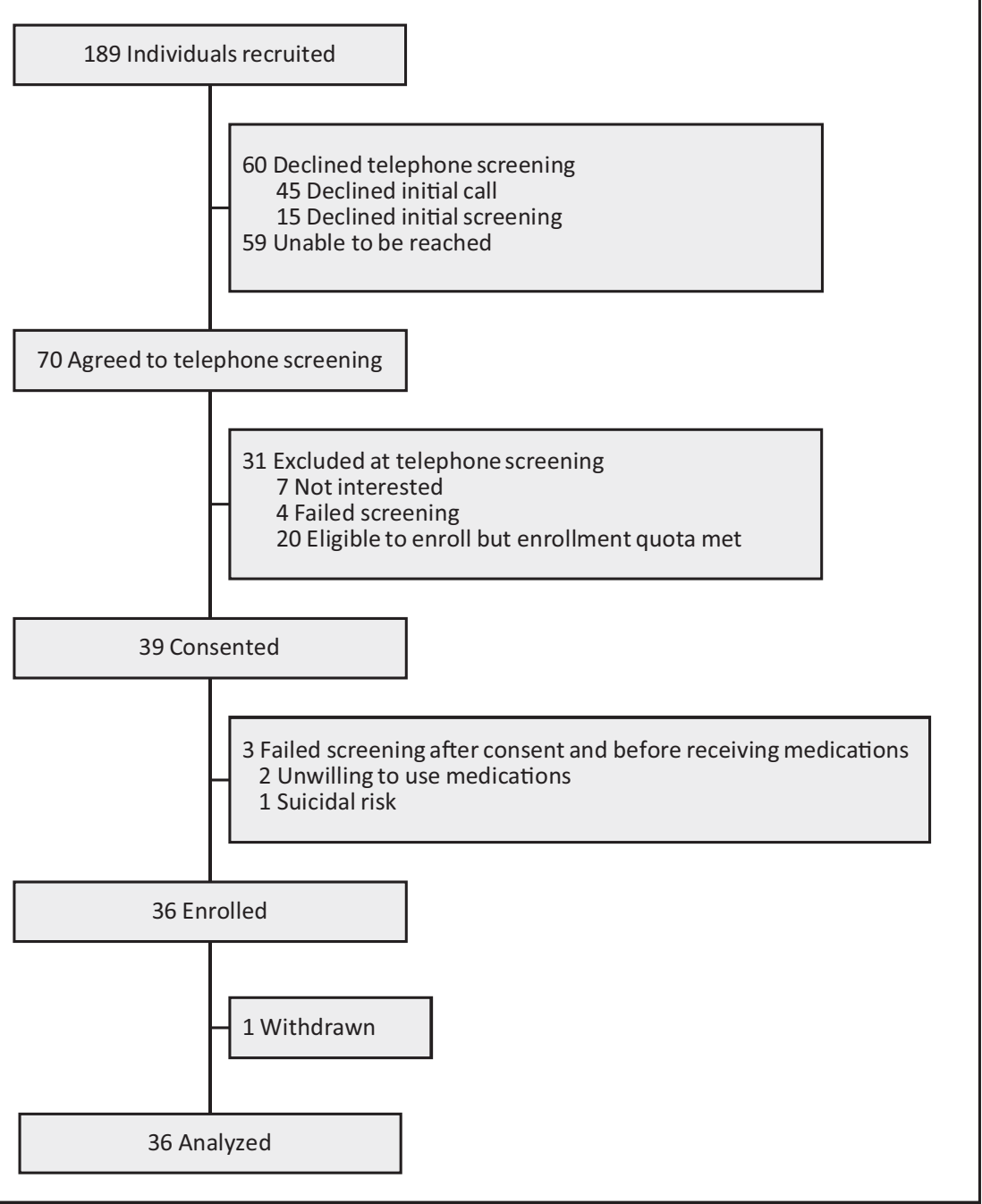

\section{Figure 1}

Flow of participants.

(see Measures section). They were evaluated medically by a study physician as to their fitness for triple therapy, based upon inclusion/exclusion criteria, baseline assessments and physical examination. If the participant was deemed medically appropriate, they were provided with study medication (see below) and one in-person counselling session as per the United States Public Health Service Clinical Practice Guideline: Treating Tobacco Use and Dependence (Fiore, Jaen and Baker, 2008). Counselling including setting a quit date, discussing previous quit attempts, planning coping strategies for urges to smoke and providing detailed information on possible AEs related to use of the study medications. Participants were asked to access a Web-based cessation support program (www.smokefree.gov) and contact the Wisconsin Tobacco Quit Line (1-800-QUITNOW) for additional behavioural therapy.

Target quit date (TQD) for all participants was set for 8 days from initiation of medications to allow for normal up-titration of varenicline. Participants were followed over the 13-week course of treatment via nine telephone calls (TQD, weeks 1, 2, 3, 4, 6, 8, 10, 12). These calls included a structured assessment of nicotine withdrawal, tolerability, treatment adherence, smoking, use of non-combustible nicotine and treatment satisfaction (see Measures section). Participants were contacted by a study physician during the 2 weeks following their TQD; all other assessments were conducted by study staff. Participants received one payment up to $\$ 140$ at Week 12 , depending on visit attendance: $\$ 50$ for orientation, $\$ 10$ for each call taken, up to nine total calls.

\section{Study Medications}

All participants received the same three medications (varenicline, nicotine patch and nicotine mini lozenge). The nicotine patch dose was based on number of cigarettes smoked daily. Specifically, participants received: 
1. Varenicline $0.5 \mathrm{mg}$ once daily for 3 days; followed by $0.5 \mathrm{mg}$ twice daily for 4 days; followed by $1 \mathrm{mg}$ twice daily for 11 weeks.

2. Transdermal Nicotine $21 \mathrm{mg}$ for 8 weeks (starting on the TQD); followed by transdermal nicotine $14 \mathrm{mg}$ for 2 weeks; followed by transdermal nicotine $7 \mathrm{mg}$ for 2 weeks (Note: for participants smoking 5-9 cigarettes/ day at baseline, the regimen was $14 \mathrm{mg} /$ day for 10 weeks followed by $7 \mathrm{mg} /$ day for 2 weeks).

3. Nicotine mini lozenges ( $2 \mathrm{mg}$ ) used as needed for relief of withdrawal and craving, for 12 weeks (starting on the TQD). Participants were urged to use at least four mini lozenges per day, but no more than 20 per day.

\section{Measures}

Baseline measures included smoking history, Fagerstrom Test of Nicotine Dependence (Heatherton, Kozlowski, Frecker, \& Fagerstrom, 1991), Wisconsin Inventory of Smoking Dependence Motives (Piper et al., 2004), Minnesota Nicotine Withdrawal Scale (Hughes \& Hatsukami, 1986), and an assessment of non-combustible tobacco use. Each structured follow-up call assessed the following outcome measures: nicotine withdrawal (Minnesota Nicotine Withdrawal Scale); non-combustible tobacco use; use of smokefree.gov and the Wisconsin Tobacco Quit Line; daily smoking (timeline follow-back to the previous call); AE assessment; satisfaction with study medications' control of withdrawal symptoms (10 point Likert scale); satisfaction with study medications' ability to help participants quit smoking (10 point Likert scale); average daily nicotine lozenge use; and treatment adherence (continued use as directed, reduced use or discontinued use) for all three study medications. The AE assessment specifically queried the presence or absence of: chest tightness, angina, dizziness, dreams, insomnia, mood changes, nausea, skin rash, sweating, dyspnea (shortness of breath) and vomiting. If present, the severity of each AE was graded as: mild (no interference with daily activities, no medications needed to treat symptom); moderate (some interference with daily activities, or over-the-counter medications needed); severe (unable to perform daily activity or prescription medication needed). Point prevalence abstinence and prolonged abstinence were assessed at 12 weeks (see Analysis for definitions).

\section{Tolerability and Safety}

The AE assessment screened for critical indicators of potential risks related to study medication, including cardiac problems and suicidal ideation. If AEs were endorsed as unresolved or new, a study physician contacted the participant to further evaluate the symptoms, study medication use and other relevant information. All unresolved AEs which may have been related to study medication were evaluated to determine if any adjustment (modification of dose or discontinuation of a medication) was needed. Any Serious Adverse Events (SAE; death or hospitalisa- tion) were to be reported to the UW Health Sciences IRB and the National Cancer Institute (study sponsor). In addition to the scheduled study assessments, participants were able to initiate contact with study staff if they had questions/concerns regarding their treatment. Study staff reviewed follow-up data on a weekly basis. UW-CTRI's standing Data Safety and Monitoring Board reviewed overall study data after the first five participants had completed their first week post-quit; after the first 20 participants had reached that milestone; and at the completion of the study.

\section{Analysis}

This was an exploratory study examining the tolerability and feasibility of combining varenicline and cNRT without treatment blinding, placebo controls or random assignment of participants. Data regarding tolerability, satisfaction and abstinence rates are reported in a descriptive manner. Point prevalence abstinence was defined as participant-reported not smoking in the 7 days prior to the week 12 follow-up call. Prolonged abstinence was defined as participant-reported continuous abstinence from Week 2 through Week 12, provided the participant was contacted on Week 12. If they smoked at all between Week 2 through Week 12, or had incomplete data, they were assumed smoking for the prolonged abstinence variable.

\section{Results}

As shown in (Figure 1), a total of 39 potential participants were enrolled in the study; three were disqualified on screening during their initial study visit, and one withdrew from the study prior to using any study medications (for reasons unrelated to the treatment). Thirty-six participants were analysed for this report, including one who withdrew at Week four, and nine participants who were unable to be reached at Week 12. Participant characteristics and smoking-related variables are shown in (Table 1). Participants had smoked for an average of 26.8 years $(S D=$ 13.9), and had previously tried quitting nearly four times $(\mathrm{M}=3.9, \mathrm{SD}=3.3)$.

\section{Tolerance}

Over the course of 13 follow-up telephone calls, 35 of 36 participants reported at least one AE. The most commonly reported AEs were insomnia, abnormal dreams and nausea. Most AEs (84\%) were mild to moderate in severity. See (Table 2) for AE prevalence and severity.

There were 20 participants $(56 \%)$ who adjusted their medications, some of whom adjusted more than one medication, or made multiple adjustments to the same medication. Six participants $(17 \%)$ decreased at least one medication but made no further changes (all AErelated), five participants (14\%) decreased at least one medication prior to discontinuing it (all AE-related), and 13 participants $(36 \%)$ discontinued at least one medication without prior dose adjustments (6 AE-related, 


\begin{tabular}{|c|c|}
\hline \multicolumn{2}{|c|}{$\begin{array}{l}\text { Participant demographics * Note, past therapeutics percentages do } \\
\text { not add to } 100 \% \text { because some participants had previously tried } \\
\text { multiple modalities }\end{array}$} \\
\hline Characteristic & $N=36$ \\
\hline Age (mean, SD) & $44(12.8)$ \\
\hline \multicolumn{2}{|l|}{ Gender $(n, \%)$} \\
\hline Male & $18(50 \%)$ \\
\hline Female & $18(50 \%)$ \\
\hline \multicolumn{2}{|l|}{ Race $(n, \%)$} \\
\hline White & $33(94 \%)$ \\
\hline Black & $1(3 \%)$ \\
\hline Asian & $1(3 \%)$ \\
\hline \multicolumn{2}{|l|}{ Marital status $(n, \%)$} \\
\hline Married or living with partner & $18(51 \%)$ \\
\hline Divorced, widowed & $9(26 \%)$ \\
\hline Never married & $8(23 \%)$ \\
\hline \multicolumn{2}{|l|}{ Annual household income $(n, \%)$} \\
\hline Less than $\$ 34,999$ & $13(38 \%)$ \\
\hline$\$ 35,000-\$ 74,999$ & $9(27 \%)$ \\
\hline Over $\$ 75,000$ & $12(35 \%)$ \\
\hline FTND (mean, SD) & $5.4(2.4)$ \\
\hline Cigarettes per day (mean, SD) & $18(9.6)$ \\
\hline \multicolumn{2}{|l|}{ Prior quit history $(n, \% *)$} \\
\hline \multicolumn{2}{|l|}{ Longest quit attempt } \\
\hline Less than 1 day & $2(6 \%)$ \\
\hline 1-14 days & $15(42 \%)$ \\
\hline 15-30 days & $2(6 \%)$ \\
\hline $1-6$ months & $9(25 \%)$ \\
\hline $7-12$ months & $3(8 \%)$ \\
\hline More than 1 year & $5(14 \%)$ \\
\hline \multicolumn{2}{|l|}{ Past therapeutics* } \\
\hline Nicotine replacement therapy & $26(72 \%)$ \\
\hline Bupropion & $7(19 \%)$ \\
\hline Varenicline & $11(31 \%)$ \\
\hline Other (E-cigarettes, counselling, reduction, other) & $42(>100 \%$ \\
\hline
\end{tabular}

7 AE-unrelated), (see Figure 2). Of the AEs cited, nausea (seven participants), insomnia (seven participants) and vivid dreams (seven participants), followed by mood changes (six participants) were the most common reasons for medication changes. AE-unrelated reasons for discontinuing medications included relapse $(n=2)$, running out $(n=1)$, not feeling the need for medications $(n=1)$, losing medications $(n=1)$ and for no known reason $(n=6)$.

\section{Safety}

Cardiovascular symptoms, mood changes and SAEs were of particular interest. No SAEs occurred during this study.
Four participants noted mild chest tightness, one of whom decreased their nicotine patch dose; but this medication change was also related to severe dreams and moderate nausea. One participant noted moderate angina and severe dyspnea, but no changes were made to the medication regimen for these symptoms, as these symptoms pre-dated study medication use and the participant did not want to change medications. Four other participants reported dyspnea (mild to moderate), but no medication changes were deemed necessary based on symptom descriptions and participant preferences. Fourteen participants reported mood changes, 3 of the 14 reported severe mood changes, and all three discontinued medications because of the mood changes. Two discontinued varenicline and one discontinued both varenicline and the nicotine patch. Other severe AEs and associated medication changes were: vivid dreams (six participants: two discontinued varenicline, one decreased the nicotine patch), insomnia (four participants: one decreased the nicotine patch, one discontinued varenicline), nausea (three participants: one decreased the nicotine patch and varenicline, one discontinued the nicotine patch and varenicline), dizziness (two participants: two discontinued varenicline and the nicotine patch), rash (two participant discontinued the nicotine patch), and sweating (two participant discontinued varenicline but cited mood, insomnia and dreams as primary reason for this change).

\section{Satisfaction and Abstinence Rates}

Overall, participants were highly satisfied with the medications' abilities to control their withdrawal symptoms, ranking their TQD satisfaction at 7.1 $(S D=2$; Likert scale $1-10)$, and their Week 12 satisfaction at $9.1(S D=1.2)$. They were equally satisfied with the medications' abilities to help them quit smoking, ranking TQD satisfaction at $7.9(S D=2$; Likert scale $1-10)$, and their Week 12 satisfaction at $9.2(S D=1.4)$. The self-reported point-prevalence abstinence at Week 12 was 58\%, assuming that any withdrawn participants $(n=1)$ or participants with missing data $(n=9)$ had returned to smoking. Fourteen participants $(38 \%)$ reported prolonged abstinence from Week 2 through Week 12.

\section{Discussion}

Despite being a small, observational study, participants using triple smoking cessation pharmacotherapy comprising varenicline, nicotine patch and nicotine mini-lozenge, achieved a high self-reported point-prevalence abstinence rate at 12 weeks after their quit date, and rated their satisfaction with the medication regimen quite favourably. However, participants reported high rates of certain AEs; such AEs led over half the sample to alter their medication regimens. Insomnia, vivid dreams and nausea were most commonly reported, and are known side effects of 
Table 2

Adverse events incidence

\begin{tabular}{|c|c|c|c|c|}
\hline Adverse Event & $\begin{array}{l}\text { Total Participants } \\
\text { Affected }(n, \%)\end{array}$ & $\begin{array}{l}\text { Affected } \\
\text { Participants } \\
\text { Reporting Symptom } \\
\text { as Mild ( } n, \%)\end{array}$ & $\begin{array}{l}\text { Affected } \\
\text { Participants } \\
\text { Reporting Symptom } \\
\text { as Moderate }(n, \%)\end{array}$ & $\begin{array}{l}\text { Affected } \\
\text { Participants } \\
\text { Reporting Symptom } \\
\text { as Severe }(n, \%)\end{array}$ \\
\hline Insomnia & 27 (75\%) & $9(33 \%)$ & $14(52 \%)$ & $4(15 \%)$ \\
\hline Vivid dreams & $26(72 \%)$ & $7(27 \%)$ & $13(50 \%)$ & $6(23 \%)$ \\
\hline Nausea & $23(64 \%)$ & $10(43 \%)$ & $10(43 \%)$ & $3(13 \%)$ \\
\hline Mood changes & $15(42 \%)$ & $8(53 \%)$ & $4(27 \%)$ & $3(20 \%)$ \\
\hline Dizziness & $14(39 \%)$ & $8(57 \%)$ & $4(29 \%)$ & $2(14 \%)$ \\
\hline Skin Rash & $7(19 \%)$ & $5(71 \%)$ & $1(14 \%)$ & $1(14 \%)$ \\
\hline Sweating & $7(19 \%)$ & $4(57 \%)$ & $2(29 \%)$ & $1(14 \%)$ \\
\hline Shortness of breath & $5(14 \%)$ & $3(60 \%)$ & $1(20 \%)$ & $1(20 \%)$ \\
\hline Vomiting & $5(14 \%)$ & $4(80 \%)$ & $1(20 \%)$ & - \\
\hline Chest tightness & $4(11 \%)$ & $4(100 \%)$ & - & - \\
\hline Angina & $1(3 \%)$ & - & $1(100 \%)$ & - \\
\hline
\end{tabular}

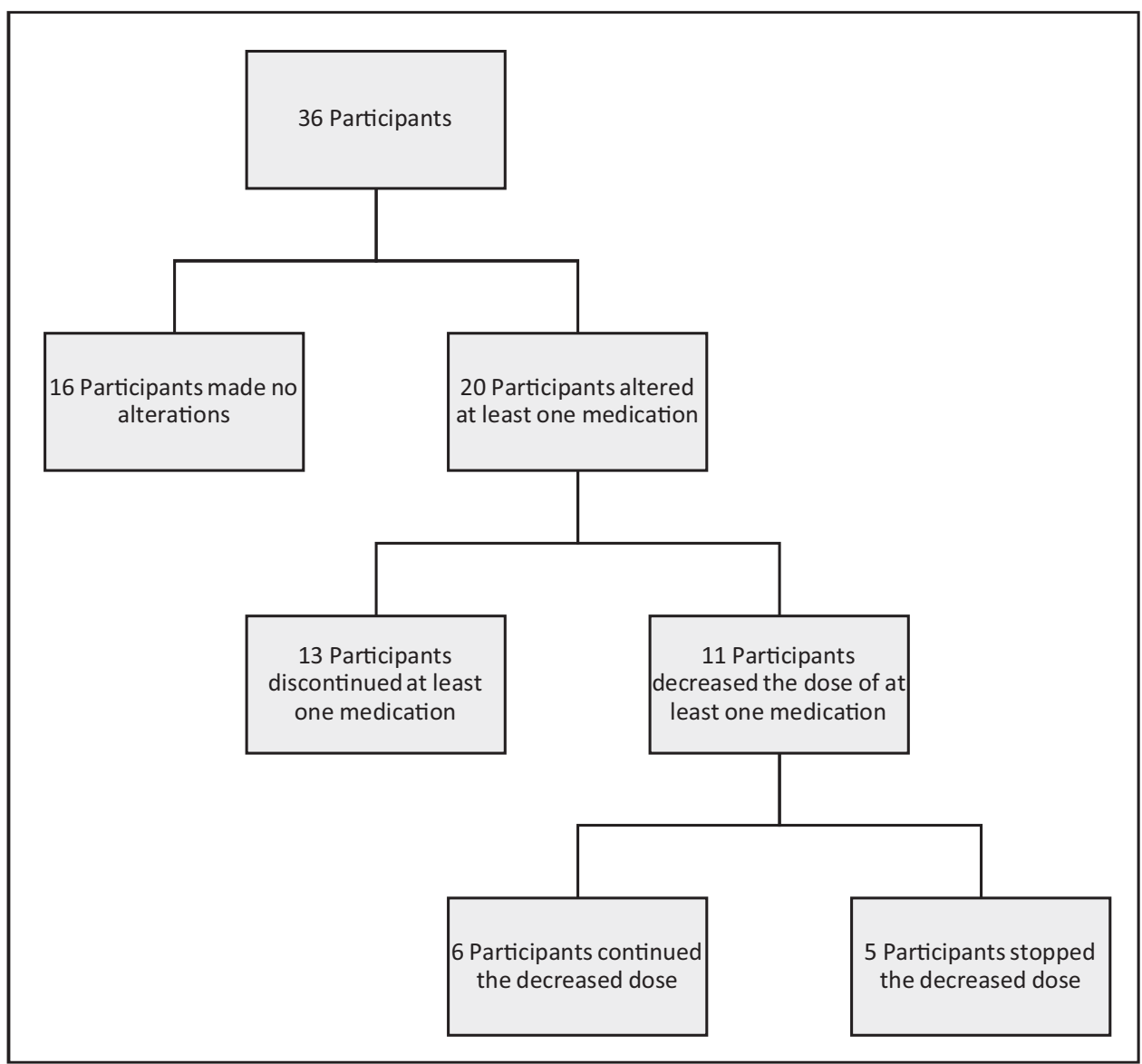

Figure 2

Flow chart of medication alterations. 
both cNRT and varenicline. The rate of reported AEs in this study was considerably more than previously reported with relevant monotherapies or combination therapies (Chang et al., 2015; Ebbert, Burke, Hays, \& Hurt, 2009). This could be due to our assessment methodology. In this study, we asked explicitly about each AE during each follow-up telephone encounter. Prior studies typically did not indicate whether they queried the occurrence of specific AEs (Hajek, Smith, Dhanji, \& McRobbie, 2013; Koegelenberg et al., 2014; Ramon, Morchon, Baena, \& Masuet-Aumatell, 2014). In short, while inter-study comparisons are difficult, we do know that in this study of triple medications, both $\mathrm{AE}$ reports and medication adjustment were common.

While 20 participants $(56 \%)$ changed their medications, five of these altered their medications for reasons unrelated to AEs. Thus, 15 participants $(42 \%)$ changed their medications due to an AE. Despite these medication changes and rates of AEs, our study completion rate was $72 \%$. This surpasses two of the three previous varenicline plus nicotine patch studies (Hajek, Smith, Dhanji, \& McRobbie, 2013; Koegelenberg et al., 2014) and is on par with the third study (Ramon, Morchon, Baena, \& Masuet-Aumatell, 2014). It is possible that the participant reimbursement may have contributed to retention rate; however, participant satisfaction rates speak against retention solely due to financial gains. Participants also self-selected to be in an observational study examining tolerability of a new experimental, intensive drug therapy. Thus, participants may have been especially highly motivated to receive treatment and quit smoking.

There were no SAE, cardiovascular events or suicidality reported in this study. This is in-line with other studies investigating reported events from varenicline and NRT (Anthenelli et al., 2016; Cahill, Lindson-Hawley, Thomas, Fanshawe, \& Lancaster, 2016; Mills, Thorlund, Eapen, Wu, \& Prochaska, 2014). One participant in our study did report to an emergency room with complaints of nausea, dizziness, vivid dreams, impatience, frustration and anger; varenicline and nicotine patches were stopped for this participant. No suicidality or inappropriate behaviour was noted, and the participant was not admitted to hospital. We demonstrated an $8 \%$ rate of severe mood changes in this study. This is higher than other studies (Ebbert, Burke, Hays, \& Hurt, 2009; Koegelenberg et al., 2014); however, our higher event rate could be related to specifically querying participants for mood changes at every contact point. An important limitation to the reported tolerability of these medications is that participants worked with study physicians to alter medications based on AEs; it is unknown what the $\mathrm{AE}$ rate or tolerability profile would have been had patients been encouraged to stay on their medications.

Participant satisfaction rates in this study were high. At the end of treatment, 17 participants (81\%) reported a score of 9 or 10 (out of 10, Likert scale) for their satisfac- tion with the medications in helping them quit smoking and 16 participants (76\%) reported a score of 9 or 10 for their satisfaction with the medications' ability to control their withdrawal symptoms. Multiple patients volunteered that they 'love the program and the meds' because it allowed them to be able to quit, one participant in particular noting that this program was 'fundamentally different' and that this quit attempt had been relatively easy compared to previous, unsuccessful quit attempts. To the best of our knowledge, this is the first study to report patient satisfaction rates using combination nicotine plus varenicline, although rates may be affected given the participants all volunteered for a an observational study assessing tolerability of a new, intense combination medical therapy.

The self-reported point-prevalence abstinence rate at the end of treatment was high at $58 \%$, although prolonged abstinence was lower at $38 \%$. This point-prevalence rate was slightly higher than point-prevalence rates reported in other trials of varenicline plus nicotine patch (Koegelenberg et al., 2014; Ramon, Morchon, Baena, \& MasuetAumatell, 2014), and the prolonged abstinence rate in this study was on par with prior studies (Hajek, Smith, Dhanji, \& McRobbie, 2013; Koegelenberg et al., 2014; Ramon, Morchon, Baena, \& Masuet-Aumatell, 2014). The three other varenicline plus NRT trials reported varying definitions of prolonged abstinence, including: 4-week continuous abstinence at week 12 (Koegelenberg et al., 2014), continuous abstinence from week two through week 12 (Ramon, Morchon, Baena, \& Masuet-Aumatell, 2014), and continuous abstinence from the quit date through week 12, with up to five lapses allowed (Hajek, Smith, Dhanji, \& McRobbie, 2013). Koegelenberg et al. (2014) had the highest reported abstinence rates, but also had the most lenient prolonged abstinence definition, and the greatest methodologic differences from the other two studies and the current study, using nicotine patches for 2 weeks pre-quit. Importantly, all of these previous studies used biochemical confirmation for abstinence rates, while we relied on participant self-report. Thus, the lack of biochemical confirmation, the high rate of medication adjustments, and absence of a control condition make it difficult to draw any firm inferences regarding effectiveness.

This study has important limitations. As an observational study, there is no comparison or control group, limiting causal inference, and the sample size is small, limiting generalisability. These results are descriptive and need confirmation. This study had a significant participant contact burden, likely increasing the sensitivity of AE assessment. Paradoxically, this likely constrains comparison with other studies that used different assessment methods. The extent of patient contact may also have improved treatment satisfaction and abstinence rates, so positive findings on these measures cannot be attributed to the triple therapy per se. Finally, 56\% of participants altered their medications, which further challenges the 
attribution of abstinence rates to the use of three medications. It may be, in fact, that trying three medications simultaneously allowed participants to find the one or two that worked for them.

Despite these limitations, this study yields informative data on both the potential drawbacks and benefits of a little studied pharmacotherapy for smoking cessation. The chief drawbacks are potentially high levels of both AE and medication changes, possibly requiring relatively intense medical monitoring. The potential advantages could include high levels of patient satisfaction and abstinence. Additional studies with larger sample sizes, and randomisation to experimental and control conditions are needed. Another potentially important finding from this study is that intensive querying of each possible adverse event may produce meaningfully higher levels of AE than less focused assessments. It may be that many previous studies have significantly underestimated the occurrence of AE. Although participants in this study reported high rates of AE, no serious AE, cardiovascular events or increased rates of suicidality or inappropriate behaviour occurred, and patients overwhelmingly appreciated the combination medication approach and the support it gave them to stop smoking.

\section{Acknowledgements}

This work was presented at the Society of General Internal Medicine 2017 Annual Meeting in Washington, D.C. (April 19-22, 2017). The authors wish to thank Stevens S. Smith, PhD, University of Wisconsin Center for Tobacco Research and Intervention, for his help with statistical analyses.

\section{Financial Support}

Author KMB was supported by a National Research Service Award from the Health Resources and Services Administration [T32HP10010] to the University of Wisconsin Department of Family Medicine and Community Health. Authors DEJ, TBB and MCF were supported by grant 5R35CA197573 from the National Cancer Institute to the University of Wisconsin Center for Tobacco Research and Intervention. Authors TBB and MCF were also supported by grant 5P01CA180945 from the National Cancer Institute to the University of Wisconsin Center for Tobacco Research and Intervention. The funding sources did not have any role in study design, data collection, analysis or interpretation or in the writing of this report.

\section{Conflict of Interest}

Author KMB has no conflicts of interest, authors DEJ, TBB and MCF have no conflicts of interest although have received federal grants in the past 3 years.

\section{Ethical Standards}

The authors assert that all procedures contributing to this work comply with the ethical standards of the relevant national and institutional committees on human experimentation and with the Helsinki Declaration of 1975, as revised in 2008 .

\section{References}

Anthenelli, R. M., Benowitz, N. L., West, R., St Aubin, L., McRae, T., Lawrence, D. et al. (2016). Neuropsychiatric safety and efficacy of varenicline, bupropion, and nicotine patch in smokers with and without psychiatric disorders (EAGLES): A double-blind, randomised, placebo-controlled clinical trial. Lancet, 387(10037), 2507-2520.

Benowitz, N. L. (2010). Nicotine addiction. New England Journal of Medicine, 362(24), 2295-2303.

Cahill, K., Lindson-Hawley, N., Thomas, K. H., Fanshawe, T. R., \& Lancaster, T. (2016). Nicotine receptor partial agonists for smoking cessation. Cochrane Database Syst Rev, May 9; (5), CD006103.

Chang, P. H., Chiang, C. H., Ho, W. C., Wu, P.Z., Tsai, J. S., \& Guo, F. R. (2015). Combination therapy of varenicline with nicotine replacement therapy is better than varenicline alone: a systematic review and meta-analysis of randomized controlled trials. BMC Public Health, 15, 689.

Ebbert, J. O., Burke, M. V., Hays, J. T., \& Hurt, R. D. (2009). Combination treatment with varenicline and nicotine replacement therapy. Nicotine \& Tobacco Research, 11(5), 572576.

Ebbert, J. O., Hays, J. T., \& Hurt, R. D. (2010). Combination pharmacotherapy for stopping smoking: what advantages does it offer?. Drugs, 70(6), 643-650.

Fiore, M. C., Jaen, C. R., Baker, T. B. et al. (2008). Treating Tobacco Use and Dependence: 2008 Update. Clinical Practice Guideline. Rockville, MD: U.S. Department of Health and Human Services. Public Health Service. May 2008.

Hajek, P., Smith, K. M., Dhanji, A. R., \& McRobbie, H. (2013). Is a combination of varenicline and nicotine patch more effective in helping smokers quit than varenicline alone? A randomised controlled trial. BMC Medical, 11, 140.

Heatherton, T. F., Kozlowski, L. T., Frecker, R. C., \& Fagerstrom, K. O. (1991). The fagerstrom test for nicotine dependence: A revision of the fagerstrom tolerance questionnaire. British Journal of Addiction, 86(9), 1119-1127.

Hughes, J. R., \& Hatsukami, D. (1986). Signs and symptoms of tobacco withdrawal. Archives of General Psychiatry, 43(3), 289-294.

Koegelenberg, C. F., Noor, F., Bateman, E. D., van Zyl-Smit, R. N., Bruning, A., O’Brien, J. A. et al. (2014). Efficacy of varenicline combined with nicotine replacement therapy vs varenicline alone for smoking cessation: a randomized clinical trial. Journal of the American Medical Association, 312(2), 155-161.

Mills, E. J., Thorlund, K., Eapen, S., Wu, P., \& Prochaska, J. J. (2014). Cardiovascular events associated with smoking cessation pharmacotherapies: A network meta-analysis. Circulation, 129(1), 28-41. 
Pfizer Laboratories Div Pfizer Inc. (2016). Chantix - varenicline tartrate tablet. 12/1/2016, from http://labeling.pfizer.com/ ShowLabeling.aspx?id $=557$

Piper, M. E., Piasecki, T. M., Federman, E. B., Bolt, D. M., Smith, S. S., Fiore, M. C. et al. (2004). A multiple motives approach to tobacco dependence: The Wisconsin Inventory of Smok- ing Dependence Motives (WISDM-68). Journal of Consulting and Clinical Psychology, 72(2), 139-154.

Ramon, J. M., Morchon, S., Baena, A., \& Masuet-Aumatell, C. (2014). Combining varenicline and nicotine patches: A randomized controlled trial study in smoking cessation. $B M C$ Medical, 12, 172. 